\title{
Analytic continuation in QCD-like theories at finite density and finite isospin
}

\author{
P. Cea \\ Università di Bari \& INFN - Bari, Italy \\ E-mail: paolo.cea@ba.infn.it \\ L. Cosmai* \\ INFN - Bari, Italy \\ E-mail: leonardo.cosmai@ba.infn.it \\ M. D’Elia \\ Università di Genova \& INFN - Genova, Italy \\ E-mail: massimo.delia@ge.infn.it
}

\section{A. Papa}

Università della Calabria \& INFN - Cosenza, Italy

E-mail: papa@cs.infn.it

\begin{abstract}
The method of analytic continuation is one of the most powerful tools to circumvent the sign problem in lattice QCD. The present study is part of a larger project which, based on the investigation of QCD-like theories which are free of the sign problem, is aimed at testing the validity of the method of analytic continuation and at improving its predictivity, in view of its application to real QCD. We have shown that a considerable improvement can be achieved if suitable functions are used to interpolate data with imaginary chemical potential. We present results obtained in a theory free of the sign problem such as two-color QCD at finite chemical potential.
\end{abstract}

The XXVII International Symposium on Lattice Field Theory - LAT2009

July 26-31 2009

Peking University, Beijing, China

*Speaker. 


\section{Introduction}

The investigation the phase diagram of QCD in the temperature-chemical potential plane has a deep relevance and implications on cosmology, astrophysics and in the phenomenology of heavy ions collisions. The lattice formulation of QCD is the only tool to approach this important issue starting from first principles. Unfortunately the study of QCD at non-zero baryonic density by numerical simulations on a space-time lattice is plagued by the well-known sign problem: the fermion determinant is complex and the Monte Carlo sampling becomes unfeasible. One of the possibilities to circumvent this problem is to perform Monte Carlo numerical simulations for imaginary values of the baryonic chemical potential, where the fermion determinant is real and the sign problem is absent, and to infer the behavior at real chemical potential by analytic continuation. The method of analytic continuation [1-16] is well-founded and works fine within the limitations posed by the presence of non-analyticities and by the periodicity of the theory with imaginary chemical potential [17].

It is very important to answer the question about which is the optimal way to extract information from data taken at imaginary values of the chemical potential. This is equivalent to answer which is the best interpolating function for data at imaginary chemical potential that could analytically continued in order to get physical predictions for real values of $\mu$. The aim of our investigations in the past two years has been to to study limitations and possible improvements of the method of analytic continuation $[9,18,19]$. In order to study this problem we have considered $\mathrm{SU}(2)$ (two-color QCD) and SU(3) at finite isospin. Indeed these theories are free of the sign problem and Monte Carlo numerical simulations at real values of the chemical potential or at real values of isospin potential are feasible. Therefore it is possible to compare the analytic continuations with the data from direct simulations allowing at the same time to discriminate between interpolating functions an to test the range of the reliability of the analytic continuation. Here we briefly review results obtained in studying analytic continuation of physical observables and of the critical line in two-color QCD. Results obtained for SU(3) at finite isospin has been reviewed in ref. [20].

\section{Analytic continuation of physical observables}

As already shown long ago [17], the partition function of any $\mathrm{SU}(\mathrm{N})$ gauge theory with nonzero temperature and imaginary chemical potential, $\mu=i \mu_{I}$, is periodic in $\theta \equiv \mu_{I} / T$ with period $2 \pi / N$ and that the free energy $F$ is a regular function of $\theta$ for $T<T_{E}$, while it is discontinuous at $\theta=2 \pi(k+1 / 2) / N, k=0,1,2, \ldots$, for $T>T_{E}$, where $T_{E}$ is a characteristic temperature, depending on the theory.

We have considered SU(2) in presence of $n_{f}=8$ degenerate staggered fermions of mass $a m=0.07$. Figure 1 shows the the tentative phase diagram in the $\left(\mu_{I}, \beta\right)$ plane for this theory (in correspondence of a fermion mass am $=0.07$ ) with $\beta_{E} \simeq 1.55$ [21, 22] and $\beta_{c} \simeq 1.41$ [23]. We have performed numerical simulations on a $16^{3} \times 4$ lattice using the exact $\phi$ algorithm [21] with $d t=0.01$ (typical statistics: $20 \mathrm{k}$ trajectories). We have performed a careful test of the analytic continuation of physical observables. A detailed discussion of the results obtained is reported in ref. [9]. In order to show the importance of a careful choice of the interpolating function of the imaginary chemical potential data, in figure 2 we display results for two different observables in 


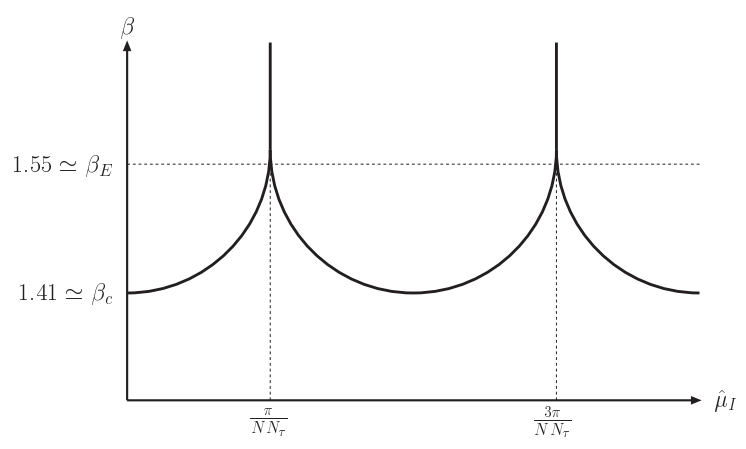

Figure 1: Phase diagram in the $\left(\beta, \hat{\mu}_{I}\right)$-plane; $N$ is the number of colors, $N_{\tau}$ the extension of the lattice in the temporal direction. The numerical values for $\beta_{E}$ and $\beta_{c}$ are valid for SU(2) in presence of $n_{f}=8$ degenerate staggered fermions with mass $a m=0.07$

correspondence of two different values of $\beta$. We have shown that the use of ratio of polynomials as interpolating function can lead to a dramatic improvement in the analytic continuation of physical observables.

The aim of our subsequent investigations has been to understand if a careful choice of the interpolating function can also improve the continuation of the critical line.
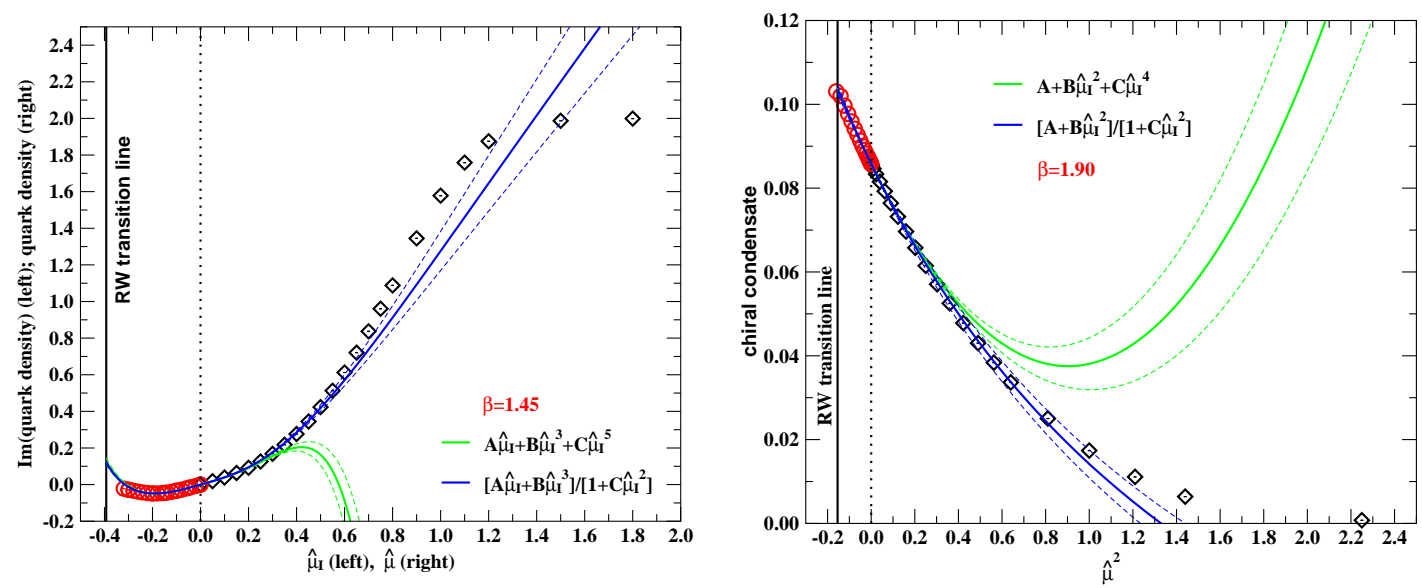

Figure 2: (Left) Negative side of the horizontal axis: imaginary part of the fermion number density $v s$. the imaginary chemical potential at $\beta=1.45$. Positive side of the horizontal axis: real part of the fermion number density $v s$. the real chemical potential at $\beta=1.45$. The green (blue) solid lines represent the polynomial (ratio of polynomials) interpolating function; the dashed lines give the corresponding uncertainty, coming from the errors in the parameters of the fit. (Right) Chiral condensate vs. $\mu^{2}$ at $\beta=1.90$. The green (blue) solid lines represent the polynomial (ratio of polynomials) interpolating function; the dashed lines give the corresponding uncertainty, coming from the errors in the parameters of the fit.

\section{Analytic continuation of the critical line}

The determination of the critical line in the $(T, \mu)$ plane is of overwhelming importance for the study of strong interactions at finite temperature and baryon density. The analytic continuation of the (pseudo-)critical line on the temperature-chemical potential plane is well-justified, but a careful 


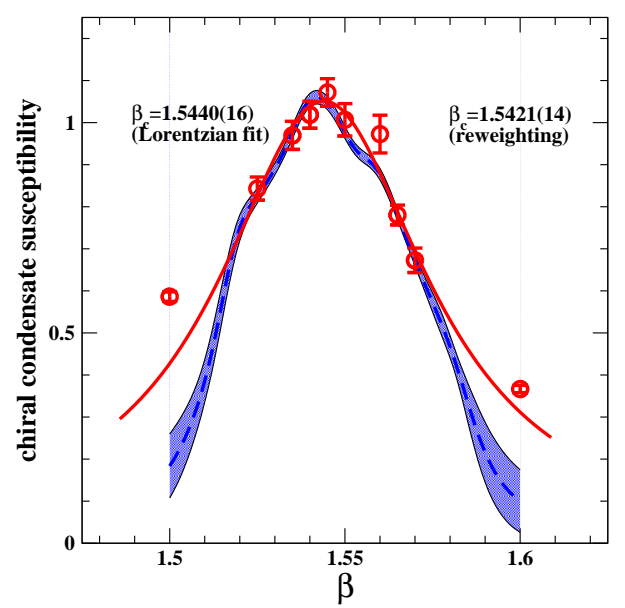

Figure 3: Chiral susceptibility at $(a \mu)^{2}=-0.1225$ vs. $\beta$. Full red line is the Lorentzian fit. Dashed blue line is the multihistogram reweighting within its bootstrap error (blue strip).

test in two-color QCD and three-color QCD with finite isospin chemical potential has cast some doubts on its reliability [18-20].

\begin{tabular}{rrrrrrr}
\hline \hline$(a \mu)^{2}$ & $\langle\bar{\psi} \psi\rangle$ & $\chi^{2} /$ d.o.f. & $\langle L\rangle$ & $\chi^{2} /$ d.o.f. & $\langle P\rangle$ & $\chi^{2} /$ d.o.f. \\
\hline-0.1225 & $1.5440(16)$ & 1.34 & $1.5349(43)$ & 0.85 & $1.5418(24)$ & 0.93 \\
-0.09 & $1.5068(15)$ & 0.65 & $1.5019(29)$ & 0.25 & $1.5046(21)$ & 1.06 \\
-0.0625 & $1.4775(29)$ & 0.88 & $1.4665(32)$ & 0.31 & $1.4755(37)$ & 0.65 \\
-0.04 & $1.4532(16)$ & 0.50 & $1.4453(36)$ & 0.76 & $1.4522(26)$ & 1.21 \\
-0.0225 & $1.4324(22)$ & 1.20 & $1.4240(28)$ & 0.80 & $1.4300(39)$ & 0.80 \\
-0.01 & $1.4197(16)$ & 1.86 & $1.4104(33)$ & 0.43 & $1.4199(26)$ & 1.45 \\
0. & $1.4102(18)$ & 0.07 & $1.3989(61)$ & 0.49 & $1.4117(32)$ & 0.07 \\
0.04 & $1.3528(22)$ & 2.91 & $1.3388(72)$ & 1.01 & $1.3631(46)$ & 1.16 \\
0.0625 & $1.3145(30)$ & 1.34 & $1.2976(62)$ & 0.87 & $1.3286(50)$ & 1.28 \\
0.09 & $1.2433(59)$ & 1.09 & $1.2508(62)$ & 0.98 & $1.2864(109)$ & 0.60 \\
\hline \hline
\end{tabular}

Table 1: Summary of the values of $\beta_{c}\left(\mu^{2}\right)$ obtained by fitting the peaks of the susceptibilities of chiral condensate $\langle\bar{\psi} \psi\rangle$, Polyakov loop $\langle L\rangle$ and plaquette $\langle P\rangle$ in SU(2) on a $16^{3} \times 4$ lattice with fermion mass $a m=0.07$. For each interpolation the $\chi^{2} /$ d.o.f. is given.

In this section we present our results in the determination of the critical line in two-color QCD using the method of analytic continuation. Contrary to the case of physical observables discussed in the previous section, the theoretical basis is not straightforward since it relies on the assumption that susceptibilities, whose peak signals the presence of the transition, be analytic functions of the parameters on a finite volume [1,2]. We have tested the method of analytic continuation in the case of two-color QCD and in the case of QCD ad finite isospin density [18, 19]. As for usual QCD simulations, we have determined the critical line for imaginary values of the chemical potential $\left(\mu^{2}<0\right)$ and interpolate them by suitable functions to be continued to $\mu^{2}>0$. In order to test the reliability of the analytic continuation, the prediction obtained at real $\mu$ has been compared with direct determinations of the transition line. 

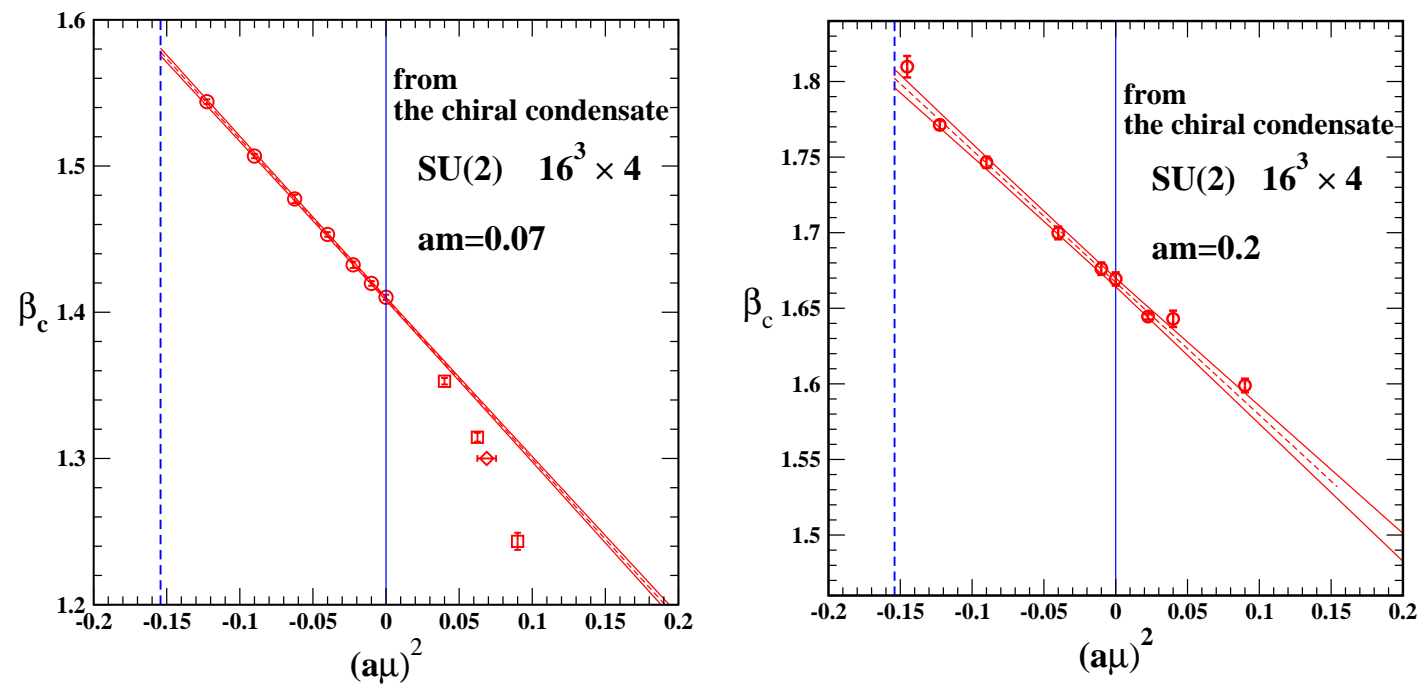

Figure 4: Critical couplings obtained from the susceptibility of chiral condensate in $\mathrm{SU}(2)$ on a $16^{3} \times 4$ lattice with $a m=0.07$ (left) and $a m=0.2$ (right), together with a linear fit (dotted line) in $(a \mu)^{2}$ to data with $\mu^{2} \leq 0$. The solid lines around the best fit line delimit the 95\% CL region.
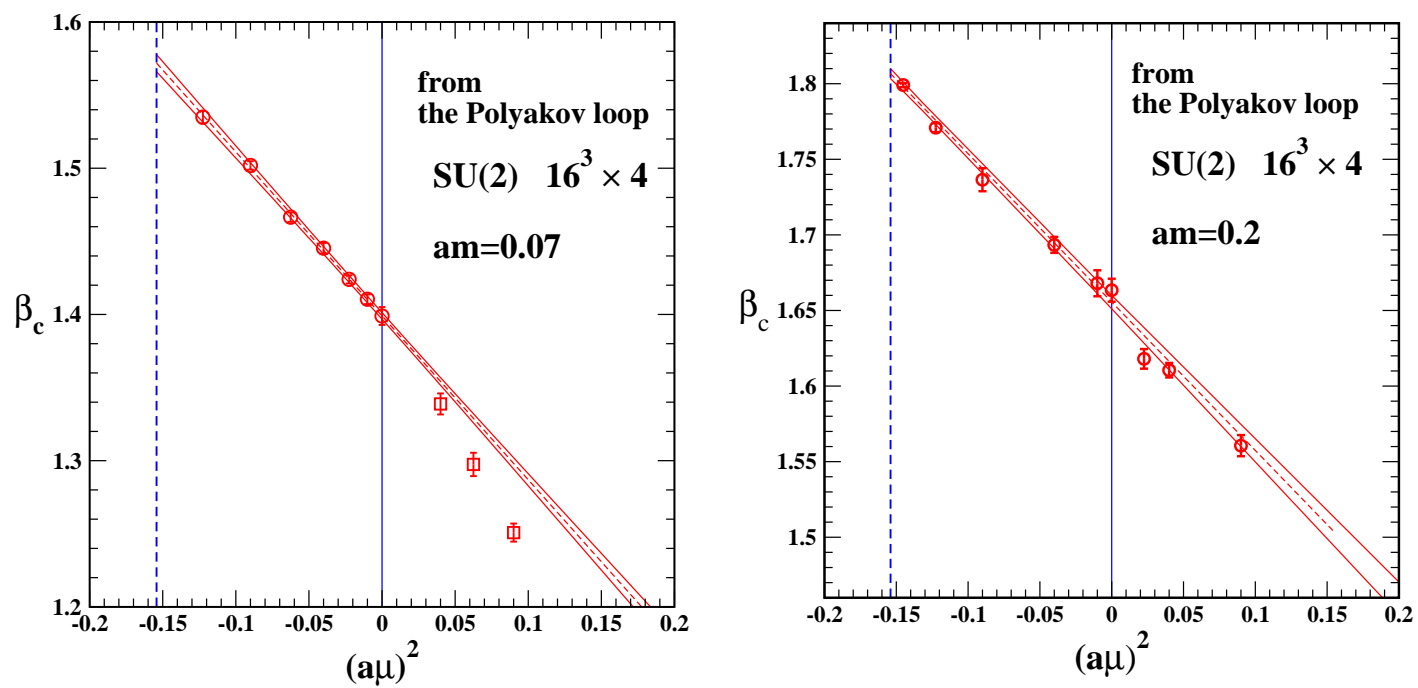

Figure 5: Critical couplings obtained from the susceptibility of Polyakov loop in SU(2) on a $16^{3} \times 4$ lattice with $a m=0.07$ (left) and $a m=0.2$ (right), together with a linear fit (dotted line) in $(a \mu)^{2}$ to data with $\mu^{2} \leq 0$. The solid lines around the best fit line delimit the 95\% CL region.

It should be remarked that on a finite volume there are no true nonanalyticities, therefore the location of the critical line may depend on the observable chosen to probe the transition. Consequently we have determined the (pseudo-)critical coupling $\beta_{c}\left(\mu^{2}\right)$ by looking at the peaks of the susceptibilities of three different observables: the chiral condensate, the Polyakov loop, and the plaquette. In figure 3 we show an example of this determination. We have fitted the peak according to a Lorentzian function. The result obtained in this way for $\beta_{c}\left(\mu^{2}\right)$ agrees well with the result obtained by means of the multihistogram reweighting.

In table 1 the values of $\beta_{c}\left(\mu^{2}\right)$ obtained in correspondence of the different "probe" observable 
(at fermion mass $a m=0.07$ ) are shown. They depend very weakly on the observable considered (only in one case the relative deviation between two determinations at the same $\mu^{2}$ slightly exceed $3 \sigma$ ). The strategy is now to interpolate the critical $\beta$ 's obtained at fixed imaginary chemical potential with an analytic function of $\mu$, to be then extrapolated to real chemical potential. For a theory free from the sign problem (such as two-color QCD) the extrapolated curve can be compared with the determinations of the critical $\beta$ 's at real chemical potential obtained through a direct computation.

As displayed on the left sides of figure 4 and figure 5, imaginary chemical potential data are very well fitted with a linear polynomial in $\mu^{2}$. If different functions are used (larger order polynomials, ratio of polynomials) the fit puts to value compatible with zero all parameters (except two of them) thus reducing again to a first order polynomial in $\mu^{2}$. This is in marked difference with what we found (see previous section and ref. [9]) for the analytic continuation of physical observables, where the ratio of polynomials performed very well. Moreover, on the left sides of figures 4 and 5 , we can clearly see a quite significative deviation between extrapolation and direct determination of the critical line at real chemical potential. The discrepancy found could imply that or the the critical line is not analytic on the whole interval of $\mu^{2}$ or that the interpolation at $\mu^{2} \leq 0$ is not accurate enough to correctly reproduce the behavior at $\mu^{2}>0$. Indeed we have found (see figure 6) that a polynomial of third order in $\mu^{2}$ nicely fits all data for $\beta_{c}\left(\mu^{2}\right)$ and therefore the critical line is analytic on the whole interval of $\mu^{2}$. A possible conclusion is that for $\mu^{2} \leq 0$ the $\mu^{4}$ and $\mu^{6}$ terms compensate each other at large negative values of $\mu^{2}$ so that the effective interpolating function of the data at $\mu^{2} \leq 0$ is a first order polynomial in $\mu^{2}$ while for $\mu^{2} \geq 0$ the $\mu^{4}$ and $\mu^{6}$ terms work in the same direction and their contribution cannot be neglected.

In order to verify if this scenario is peculiar to $\mathrm{SU}(2)$ we have investigated the same theory with a different mass $a m=0.2$ for the $n_{f}=8$ degenerate fermions. We have also examined the case of SU(3) at finite isospin [19, 20]. The reason for consider SU(2) at a larger value for the quark mass is that at any fixed temperature $T$ the critical value of $\mu$ gets larger, consequently the critical line could become less curved in the physical region $\mu^{2}>0$. Accordingly higher order terms in $\mu^{2}$ in the description of the critical line by a polynomial could be less important. We have sampled the critical line for $\mathrm{SU}(2)$ and quark mass am $=0.2$. The best interpolation of $\beta_{c}\left(\mu^{2}\right)$ data at $\mu^{2} \leq 0$ is a polynomial linear in $\mu^{2}$ and, at variance with the case of quark mass $a m=0.07$, the extrapolation to $\mu^{2}>0$ compares very well with the direct determination of $\beta_{c}\left(\mu^{2}\right)$ in that region. So we can argue that the the extrapolation to $\mu^{2}>0$ works definitely better for larger quark masses, i.e. away form the chiral limit.

\section{Conclusions}

We have reported here an investigation which is part of a larger project devoted to study the method of analytic continuation in QCD-like theories free from the sign problem and to improve its predictivity in view of its application to QCD.

For what concerns analytic continuation of physical observables we have shown that a considerable improvement can be achieved, when extrapolating data from imaginary to real chemical potentials, if ratios of polynomials are used as interpolating functions (for a thorough discussion see ref. [9]). 


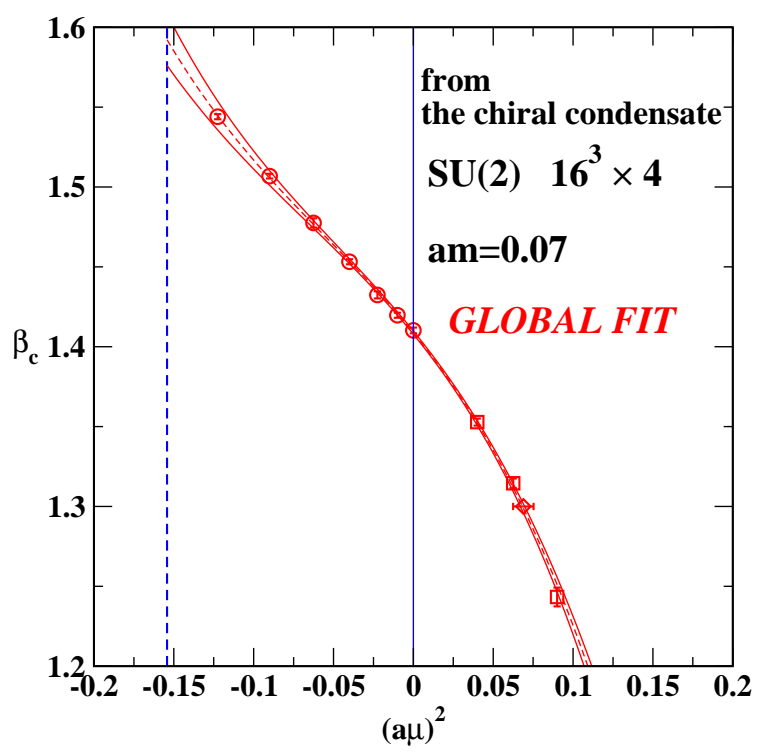

Figure 6: Same as in figure 4, but with results of a fit to all data including term up to order $\mu^{6}$.

We have also presented results for the analytic continuation of the critical line in the $(T, \mu)$ plane from imaginary to real chemical potential both in the case of two-color QCD. We have found that the critical line around $\mu=0$ can be described by an analytic function. Indeed, a third order polynomial in $\mu^{2}$ fits all the available data for the critical coupling.

We have shown that there is a clear indication that in the chiral limit high-order terms in the polynomial interpolation play a relevant role at $\mu^{2}>0$ but are less visible at $\mu^{2}<0$, this calling for extremely high accuracy in detecting such terms from simulations at $\mu^{2}<0$. The predictions for the pseudocritical couplings at real chemical potentials may be wrong if data at imaginary $\mu$ are fitted according to a linear dependence.

All the issues above have undergone further investigation in a different theory such as SU(3) at finite isospin (results for SU(3) at finite isospin are discussed in refs. [19, 20]).

The lessons we learned in studying analytic continuation in QCD-like theory free from the sign problem will be used in the near future to the determination the critical line for real QCD.

\section{References}

[1] P. de Forcrand and O. Philipsen, The QCD phase diagram for small densities from imaginary chemical potential, Nucl. Phys. B642 (2002) 290-306, [hep-lat/ 0205016$].$

[2] P. de Forcrand and O. Philipsen, The QCD phase diagram for three degenerate flavors and small baryon density, Nucl. Phys. B673 (2003) 170-186, [hep-lat/0307020].

[3] M. D'Elia and M.-P. Lombardo, Finite density QCD via imaginary chemical potential, Phys. Rev. D67 (2003) 014505, [hep-lat/0209146].

[4] M. D'Elia and M. P. Lombardo, $Q C D$ thermodynamics from an imaginary $m u(B):$ Results on the four flavor lattice model, Phys. Rev. D70 (2004) 074509, [hep-lat / 0406012 ].

[5] V. Azcoiti, G. Di Carlo, A. Galante, and V. Laliena, Phase diagram of QCD with four quark flavors at finite temperature and baryon density, Nucl. Phys. B723 (2005) 77-90, [hep-lat / 0503010]. 
[6] H.-S. Chen and X.-Q. Luo, Phase diagram of QCD at finite temperature and chemical potential from lattice simulations with dynamical Wilson quarks, Phys. Rev. D72 (2005) 034504, [hep-lat/0411023].

[7] P. Giudice and A. Papa, Real and imaginary chemical potential in 2-color QCD, Phys. Rev. D69 (2004) 094509, [hep-lat/0401024].

[8] S. Kim, P. de Forcrand, S. Kratochvila, and T. Takaishi, The 3-state Potts model as a heavy quark finite density laboratory, PoS LAT2005 (2006) 166, [hep-lat / 0510069 ].

[9] P. Cea, L. Cosmai, M. D'Elia, and A. Papa, Analytic continuation from imaginary to real chemical potential in two-color QCD, JHEP 02 (2007) 066, [hep-lat / 0612018 ].

[10] A. Papa, P. Cea, L. Cosmai, and M. D'Elia, Analytical continuation from imaginary to real chemical potential in 2-color QCD under scrutiny, PoS LAT2006 (2006) 143, [hep-lat/ 0610088 ].

[11] P. de Forcrand and O. Philipsen, The chiral critical line of $N(f)=2+1$ QCD at zero and non-zero baryon density, JHEP 01 (2007) 077, [hep-lat/ 0607017 ].

[12] P. de Forcrand, S. Kim, and O. Philipsen, A QCD chiral critical point at small chemical potential: is it there or not?, PoS LAT2007 (2007) 178, [arXiv: 0711 . 0262].

[13] M. D’Elia, F. Di Renzo, and M. P. Lombardo, The strongly interacting Quark Gluon Plasma, and the critical behaviour of QCD at imaginary chemical potential, Phys. Rev. D76 (2007) 114509, [arXiv:0705.3814].

[14] S. Conradi and M. D'Elia, Imaginary chemical potentials and the phase of the fermionic determinant, Phys. Rev. D76 (2007) 074501, [arXiv: 0707.1987$].$

[15] P. Cea, L. Cosmai, M. D'Elia, and A. Papa, Analytic continuation of the critical line in 2-color QCD at nonzero temperature and density, PoS LAT2007 (2007) 214, [arXiv: 0710 . 2068].

[16] F. Karbstein and M. Thies, How to get from imaginary to real chemical potential, Phys. Rev. D75 (2007) 025003, [hep-th/0610243].

[17] A. Roberge and N. Weiss, Gauge theories with imaginary chemical potential and the phases of QCD, Nucl. Phys. B275 (1986) 734.

[18] P. Cea, L. Cosmai, M. D'Elia, and A. Papa, The critical line from imaginary to real baryonic chemical potentials in two-color QCD, Phys. Rev. D77 (2008) 051501, [arXiv : 0712 . 3755].

[19] P. Cea, L. Cosmai, M. D’Elia, C. Manneschi, and A. Papa, Analytic continuation of the critical line: suggestions for QCD, Phys. Rev. D80 (2009) 034501, [arXiv: 0905 . 1292].

[20] P. Cea, L. Cosmai, M. D’Elia, C. Manneschi, and A. Papa, On the analytic continuation of the critical line, PoS LAT2009 (2009) 161.

[21] S. A. Gottlieb, W. Liu, D. Toussaint, R. L. Renken, and R. L. Sugar, Fermion number susceptibility in lattice gauge theory, Phys. Rev. D38 (1988) 2888-2896.

[22] P. Giudice and A. Papa, Finite temperature 2-color QCD for real and imaginary chemical potential, Nucl. Phys. Proc. Suppl. 140 (2005) 529-531, [hep-lat/ 0409022 ].

[23] Y. Liu, O. Miyamura, A. Nakamura, and T. Takaishi, Simulation of SU(2) dynamical fermion at finite chemical potential and at finite temperature, hep-lat/0009009. 